\title{
The effect of using different types orthodontic forces upon the level of interleukin ii in the human gingival cervicular fluid (clinical study)
}

\begin{abstract}
Efficiency of orthodontic forces with different durations and strengths has long been an important issue in clinical orthodontics. However, evidence on biological markers and force levels is lacking. Hence, current randomized split mouth study design was used to evaluate the efficacy of two different orthodontic force levels on inducing initial tissue reaction by measuring the mediator of bone resorption "Interleukin 2" (IL-2). The sample consisted of ten participants selected randomly from the orthodontic department of Alexandria University according to strict selection criteria; the treatment plan for all participants required extractions of upper right and left maxillary first premolars with maximum anchorage requirements. Samples of IL-2 were collected from the distal surface of canines on both sides and analyzed using ELISA kit for human IL-2. Increase in the level of IL-2 was noticed on both control and test side being $40.2 \%$ and $67.5 \%$ respectively, in the overall change in enzymatic activity. Thus, it was more efficient at the gradual increasing force side with weekly increment showing better initial tissue reaction for bone resorption.
\end{abstract}

Volume 2 Issue 6 - 2015

Ammar M Blich,' Nazla O Tamish,' Walid A El Kenany,' Myriam A Helmy²

'Department of Orthodontic, Alexandria University, Egypt

${ }^{2}$ Department of clinical pathology, Alexandria University, Egypt

Correspondence: Ammar M Blich, Department of Orthodontic, Alexandria University, Egypt, Tel 972000000000 , Email dr_ammarblich@hotmail.com

Received: May 18, 2015 | Published: August 31, 2015

Keywords: interleukin, biomarkers, bone resorption, elisa, orthodontic forces

Abbreviations: IL-2, interleukin-2; G, gram(s); PDL, periodo0ntal ligament; IL-1, interleukin-1; PGE2, prostaglandin E2; EPO, erythropoietin; IL-2R, interleukin-2 receptor; IL-8, interleukin-8; GCF, gingival crevicular fluid; IL-6, interleukin-6; $\mathrm{TNF}-\alpha$, tumor necrosis factor- $\alpha$; TGF, transforming growth factor; THPO, thrombopoietin; MMP-8, matrix metalloproteinase-8; NiTi, nickel-titanium; BF, blastogenic factor; TCGF, T-cell growth factor; pg/ $\mu 1$, picograms per microlitre; ELISA, enzyme-linked immunosorbent assay; TRC, T-cell receptor; T-regs, regulatory T-cells; FDA, food \& drug administration; BMP, bone morphogenic proteins; OSF, osteoclast differentiation factor; M-CSF, macrophage colony-stimulating factor; RANKL, receptor activator of nuclear factor-kappa ligand; OPG, osteoprotegrin; ASL, asparate amino tranferase; ECM, extracellular matrix; TPA, transpalatal arch, TRAP, tartarate resistant acid phosphatase

\section{Introduction}

Orthodontic tooth movement needs to be efficient in order to achieve shorter treatment times, reduce iatrogenic issues and enhance patient satisfaction. Para dental tissues express extensive macroscopic and microscopic changes, when exposed to variable degrees, frequency, duration and mechanical loading. In extraction cases space closure can vary from $1 \mathrm{~mm} /$ month to extremely slow in some adult patients. ${ }^{1,2}$ Orthodontic tooth movement depends on several variables such as applied force and its physical characteristics, as well as the PDL size and biological response. ${ }^{3}$ Orthodontic force-induced stresses change and affect vascularity/blood flow of the PDL, which results in local production and release of various biomarkers, such as arachidonic acid metabolites, neurotransmitters, colony-stimulating factors cytokines (IL-2), and growth factors in turn resulting in increased cellular responses from Osteoclasts, providing a positive environment for bone deposition and resorption. ${ }^{4,5}$ Thus, the mechanical stimulus that produces maximum rate of tooth movement with minimal irreversible damage to alveolar bone, root, and PDL is considered optimal. Moreover, in orthodontics force can be continuous, intermitted, constant or declining) capable of generating maximum orthodontic tooth movement, without tissue damage, and with minimal patient discomfort. ${ }^{5-8}$ Clinically, during active orthodontic treatment, the relationship between rate of tooth movement and orthodontic force level is considered to be an essential tool on an individual basis in identifying optimal forces. ${ }^{9}$ Biomechanics for space closure needs to be based on sound evidence of biomarkers which in the past has been lacking.

The identification of and the role played by cytokines in bone remodeling is an important breakthrough in bone biology. Cytokines (Greek cyto for cell and kines for movement) are a category of lowmolecular weight $(\mathrm{mw}<25 \mathrm{kDa}$ : kilo Daltons) signaling molecules (proteins) that are widely used during cellular communication. The term cytokine comprises a large and varied family of polypeptide regulators that are created extensively all over the body by cells of different embryological basis. ${ }^{10}$ Basically, the term "cytokine" refers to the immune-modulating mediators (for example interleukins). Cytokines (such as IL-2) circulate in pico-molar, ${ }^{10-12}$ concentrations that can increase up to 1,000 -fold during infection of trauma. In contrast, hormones circulate in nano-molar (10-9)..$^{10}$ Nearly, all nucleated cells, and especially endo epithelial cells and local 
macrophages are strong producers of IL-1, IL-6, and TNF- $\alpha$. (Tumor necrosis factor), interleukin 1(IL-1), interleukin 2 (IL-2), interleukin 6 (IL-6), and interleukin 8 (IL-8) are the pro-inflammatory, while interleukins IL-4, IL-10, and IL-13 are anti-inflammatory. ${ }^{11}$

The IL-2 is a pro-inflammatory cytokine released from T-helper lymphocytes. This cytokine is involved in B-cell activation and stimulates macrophages, natural killer cells, T-cell proliferation, and osteoclast activity. IL-2 has been also involved in the stimulation of osteoclast activity in bone resorption, and also suggested to play an active role in periodontal diseases pathogenesis. ${ }^{12}$ IL-2 is also essential during development of T-cells in the thymus for the maturation of a exclusive subset of $\mathrm{T}$ cells that are called regulatory $\mathrm{T}$ cells (T-regs). After leaving the thymus, the function of T-Regs is to inhibit other T cells from identifying and reacting against "self-antigens", resulting in "autoimmune reaction". T-Regs do so by inhibiting the reacting cells from producing IL-2, ${ }^{13}$ hence; it's required for IL-2 to differentiate between self and non-self, another one of the unique features of the immune system. ${ }^{14}$

A study in orthodontics ${ }^{15}$ measured levels of interleukins 8, 2, and 6 during tooth movement and came to a conclusion that leveling and distalization of the teeth induce increase in the levels of interleukins 2, 6 , and 8 in the PDL which can be detected in Gingival Crevicular Fluids (GCF). Similarly another recent study, ${ }^{16}$ observed other biomarkers such as MMP9, TIMPs, RANKL and OPG during orthodontic tooth movement and concluded that biomarker analysis would assist in optimizing orthodontic forces. Hence, GCF is considered to be a promising and strong area of future research, because these studies have already started to offer perception into the temporal features of formative and resorptive processes in the para-dental tissues during orthodontic treatment.The alternative hypothesis (HA) of the current study was that the frequency of activation of force does affect the IL-2 expression during orthodontic space closure.

\section{Materials and methods}

Ten healthy patients ( 5 females) age $17.2 \pm 2.3$ years presenting for Orthodontic treatment at the Orthodontic Department, Faculty of Dentistry, and Alexandria University were randomly selected to participate in the study. Consent form was signed by all participating subjects and ethical approval was sought. Strict selection criteria included complete eruption of permanent dentition, no significant medical history, good oral hygiene without periodontal and gingival inflammation, no bone loss radiographically and no medications. Female patients were neither pregnant/lactating nor on contraceptives. Preceding orthodontic treatment, all participants underwent full arch scaling and were instructed to follow strict oral hygiene instructions including the correct use of toothbrush and daily rinse with $0.15 \%$ Benzidamin HCL mouth (Tantum Verde, A.C.R Angelini F, Italy). Oral hygiene instruction was reinforced during the whole period of the study.

\section{Experimental design}

Split mouth design with randomization for test quadrants was utilized. First maxillary premolars were extracted in all patients with full brackets system (Omni Roth.0.022x0.028 slot, 3M Gemini Metal Brackets, 3M Unitek Corporation, USA) initial arch wire 0.014inch Nitinol protocol for all. Following alignment 0.018inch stainless steel arch wire was placed and control side received the conventional constant force for retraction of canines with Nitinol spring, while the test quadrant received varying force of initial $75 \mathrm{gm}$ in week one, $150 \mathrm{gm}$ in week two and $300 \mathrm{gm}$ in week three and four. Anchorage was reinforced in all cased with Transpalatal arch.

\section{Sample collection of gingival crevicular fluid (GCF)}

Samples were collected from the distal aspect of maxillary canines at 4 specific timings:

1. T1: BASELINE: GCF samples were collected from all patients before canine retraction on both sides, followed by activation of coil spring with level of force $75 \mathrm{gm}$ one side and $150 \mathrm{gm}$ on the other (Figure 1a).

2. T2: 1 week after T1: The second GCF samples were collected and the level of retraction force was raised to $150 \mathrm{gm}$ on one side, while it remained constant on control side (Figure 1b).

3. T3: 1 week after T2: The 3rd GCF samples were collected, and the level of retraction force was raised to $300 \mathrm{gm}$ on test side.

\section{T4: 1 week after T3: collection of 4th samples from both sides.}

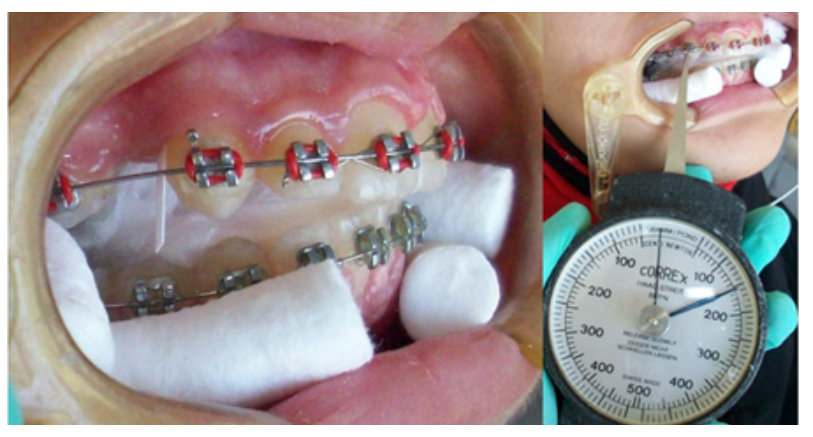

Figure I (a) Insertion of paper strips into gingival sulcus for collection of GCF at baseline. (b) Measuring the levels of force 150g on space closure site.

GCF samples were collected using paper strips (Whatman International Ltd, Maidstone, England). Each paper strip was cut and prepared with $2 \mathrm{~mm}$ in width and $10 \mathrm{~mm}$ in length according to the specification of samples collection. ${ }^{17}$ Any paper strips that were contaminated with blood or saliva during samples collection were excluded from the study. Complete isolation was ensured with sterile cotton rolls in buccal vestibules and sterile gauze in oral cavity (Figure1). A gentle air stream was applied for 10seconds distal to the canines. 4 paper strips on each side were inserted distal to the canine, one at a time for 30 seconds each, at interval of 1 minute between each paper strips \& at a depth of $1 \mathrm{~mm}$ into the gingival sulcus according to the previous study protocol. ${ }^{18}$ Care was taken to avoid any mechanical injury to the gingival sulcus. The level of GCF in each paper strip was measured using an Endodontic ruler. Uncontaminated paper strips were placed into two different Eppendorf tubes, 2 paper strips at each tube. $100 \mu \mathrm{L}$ of $0.1-\mathrm{mol} / \mathrm{L}$ phosphate buffer saline was added using $100 \mu \mathrm{L}$ pipette to each tube and mixed thoroughly by vortex mixer for 10 seconds. ${ }^{19}$ The tubes were then centrifuged for 5 minutes at a speed of 3500rpm at room temperature. Tubes were then stored at $-20^{\circ} \mathrm{C}$. The Department of General Pathology, Alexandria University till analyzed. 


\section{IL-2 level assessment in the GCF}

An enzyme-linked immuno sorbent assay (ELISA) kit (Immulite, Diagnostic products, Los Angeles, Calif, USA) specific for human IL-2 which didn't cross-react with other cytokines was used to determine the level of IL-2 in each tube. Total amount of IL-2 was measured in picograms $/ \mu \mathrm{L}(\mathrm{pg} / \mu \mathrm{L})$.

\section{Statistical evaluation}

Wilcoxon signed ranks test was used to determine statistical difference between and among the groups. Total change in Interleukin-2 activity throughout the entire experimental period was assessed by the amount of change in the enzymatic level at each point (T2-T3\&T4) after initiation of canine retraction is compared to baseline value (T1) Both control and test groups was calculated using the following equation:

$$
\mathrm{Z}=(\mathrm{X}-\mathrm{Y}) / \mathrm{Y} * 100
$$

$Z$ : Amount of change in enzyme level

$\mathrm{X}$ : Enzyme level at a certain point (after initiation of canine retraction)

Y: Baseline enzyme level

After calculating the percentage of change at each point, overall means $\left(Z^{1}, Z^{2}, Z^{3} \ldots Z^{1} 0\right)$ were calculated to assess the total change in Interleukin-2 levels at each sampling site.

\section{Results \& discussion}

\section{Results}

All ten subjects completed the study and maintained good oral hygiene throughout the experiment. Descriptive statistics (mean, standard deviation (SD), minimum and maximum values) for both study groups are presented in Table 1 . The concentrations of IL-2 at T1, T2, T3 and T4 from both sides for each patient participated in this study are given in Table 2. Significance was set at less than 0.05 $(\mathrm{P} \leq 0.05)$. The level of IL-2 is significantly higher at $\mathrm{T} 3(\mathrm{p}=0.02)$ and progressively stays higher at $\mathrm{T} 4$ albeit not as significant as T3. It is clear that the overall change in the enzymatic activity compared to baseline, enzyme levels in the relatively constant force group(control group) revealed an increase in the activity by $40.23 \%$ while at the gradually increasing force(Test group) the increase was $67.5 \%$. It is evident that the overall increase in the IL-2 activity in the gradually increasing force group is greater than that in the relatively constant force group which is statistically significant in Figure 2-4. Measurements at each time interval were compared with successive follow up week in each group; no significant increase was seen in the test group while the control group showed significant increase in IL-2 levels between T1 and $\mathrm{T} 2$, and also between $\mathrm{T} 3$ and $\mathrm{T} 4$.

Table I Comparison of IL-2 level between both groups at baseline and different follow up periods:WSR test:Wilcoxon signed ranks test. The only significant difference between both groups was found at after two weeks T3

\begin{tabular}{|c|c|c|c|c|}
\hline \multirow{3}{*}{ Follow up Periods } & \multicolumn{4}{|l|}{ Interleukin 2 Mean \pm SD } \\
\hline & \multirow[b]{2}{*}{ Constant Force Teeth } & \multirow[b]{2}{*}{ Changing Force Teeth } & \multirow[t]{2}{*}{ WSR Test } & \multirow[t]{2}{*}{ P Value } \\
\hline & & & & \\
\hline Baseline "TI" & $4.39 \pm 3.02$ & $4.62 \pm 3.41$ & 0.31 & 0.76 \\
\hline After one week "T2" & $5.74 \pm 3.5 \mathrm{I}$ & $6.53 \pm 5.28$ & 0.56 & 0.58 \\
\hline After two weeks "T3" & $4.98 \pm 3.97$ & $6.29 \pm 3.70$ & 2.31 & $0.02 *$ \\
\hline After three weeks "T4" & $6.02 \pm 2.96$ & $6.63 \pm 2.49$ & 1.78 & 0.08 \\
\hline
\end{tabular}

*: Statistically significant at $\mathrm{P} \leq 0.05$

Table 2 Overall change in IL-2 levels throughout the entire experimental period at the 2 sampling sites

Overall Change in IL-II Levels*

\begin{tabular}{llll}
\hline \multicolumn{2}{l}{ Relatively Constant Force Group } & \multicolumn{2}{l}{ Gradually Increasing Force Group } \\
\hline \multicolumn{2}{l}{ Control Group } & \multicolumn{2}{l}{ Test Group } \\
\hline$Z_{1}$ & $15.16 \%$ & $Z_{1}$ & $43.18 \%$ \\
$Z_{2}$ & $38.36 \%$ & $Z_{2}$ & $-1.4 \%$ \\
$Z_{3}$ & $-6.07 \%$ & $Z_{3}$ & $11.21 \%$ \\
$Z_{C}$ & $40.23 \%$ & $Z_{T}$ & $67.5 \%$
\end{tabular}




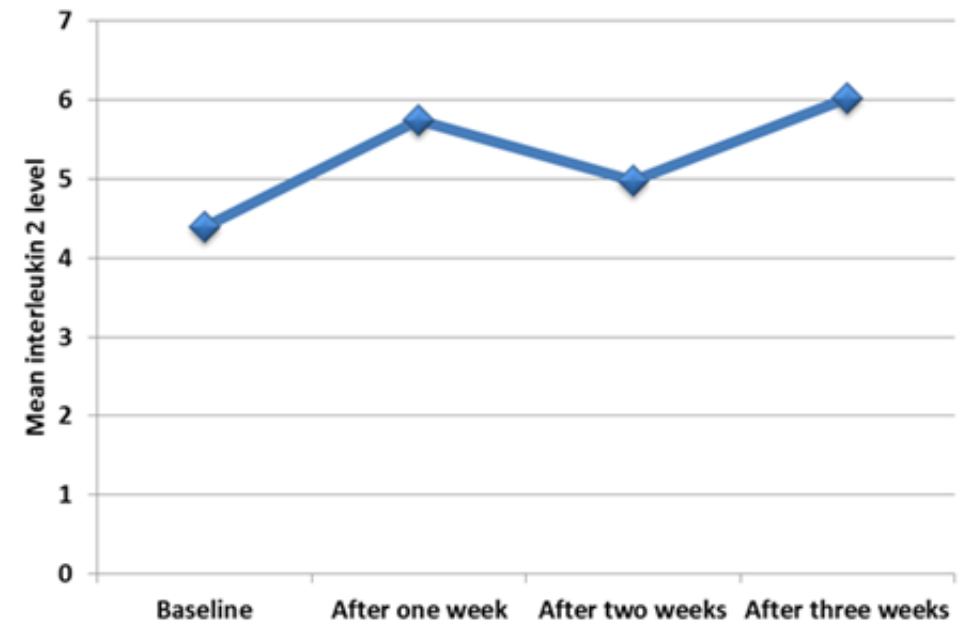

Figure $\mathbf{2}$ Line graph shows the changing in IL-2 level at different periods using constant level of force.

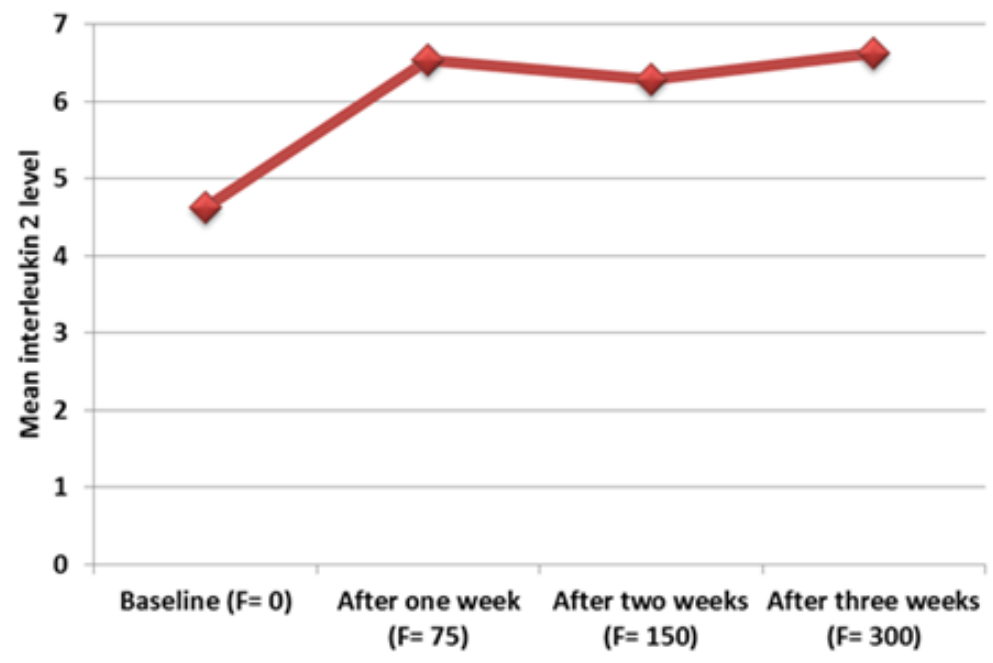

Figure 3 Graph shows the changing in IL-2 level at different periods using changing level of force.

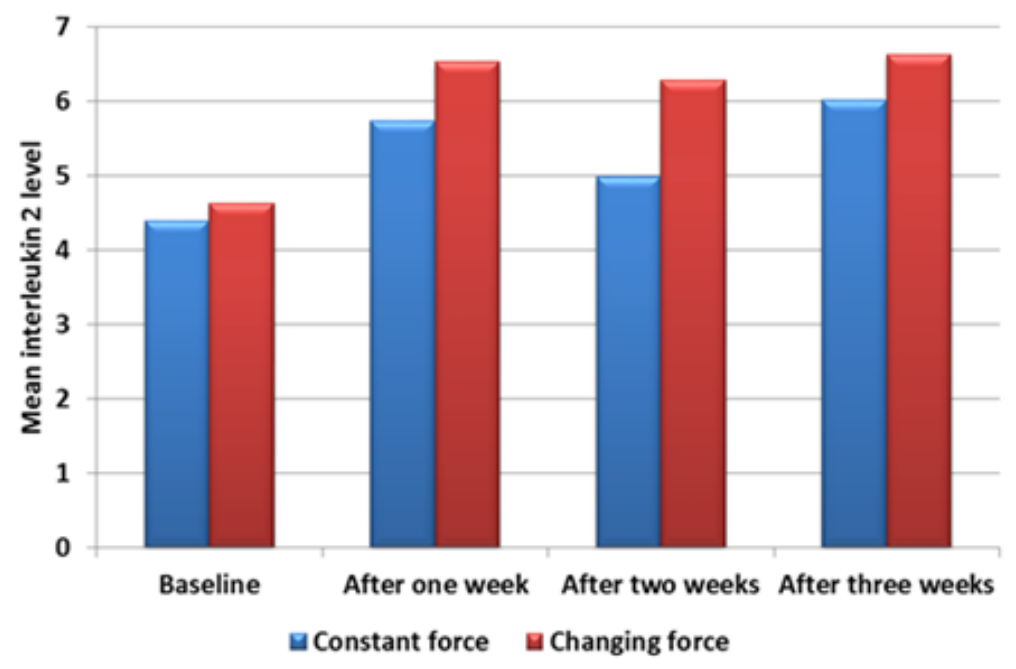

Figure 4 Bar graph shows the changing in IL-2 level in both groups together. 


\section{Discussion}

The evidence for the optimal force level and duration for efficient tooth movement in Orthodontics has been lacking. To the best of knowledge of the authors there has been no previous work on ascertaining the optimal activation time with scientific evidence related to IL-2 levels in humans, previous work in this field is mostly done on animal model. ${ }^{20}$ Hence, the present study has made an effort to assess the effectiveness of using two different types of orthodontic forces on prompting initial tissue response by measuring IL-2 as a biomarker of bone resorption. Cytokines play a key role in bone remodeling, ${ }^{21}$ but measuring levels during active tooth movement with varying force levels provides the optimal activation time frame. GCF analysis is a valuable technique, particularly for the human studies in vivo, as it is non-invasive allowing sequential sampling. The premise of the current experimental study is based on the ability to predict PDL compression resulting from migration of biochemical products into the gingival sulcus.

Comparing in-situ methods for GCF biochemical analysis, involved sampling of crevicular fluid using either paper strips or the microcapillary tubules. ${ }^{4}$ In current study paper-strips method was employed instead of micro-capillary tubules because the later may disrupts the crevicular epithelium and results in GCF contamination with blood and serum. Thus, using paper strips of proper size (10x8) with careful insertion avoids contamination of samples. ${ }^{17}$ Moreover, the collection site is of interest too, ${ }^{22}$ in the current study it was standardized to canine only with IL-2 samples collected from the distal surface of maxillary canines as it was expected that bone resorption would predominate at the distal side of the maxillary canines during space closure. Maintaining a good oral hygiene was essential to minimize the effect of gingival inflammation on cytokines level. Hence, all the participants were given proper instructions on oral hygiene along with regular use of toothbrush and a mouthwash. In a study conduct by Serra et al., ${ }^{23}$ indicates that gender and age did not rise enzymatic activity therefore our study did not have age/gender restrictions.

The issue with force decay is another variable which needs to be kept constant. Previous work, ${ }^{24}$ determined the force degradation of closed coil springs made up of stainless steel (SS), Cobalt-ChromiumNickel and Nickel-Titanium alloys. The springs were extended to the original extension at intervals of 4hours, 24hours, 3days, 7days, 14days, 21days and 28days and the level of force generated was recorded. The final conclusion was that NiTi coil springs were advantageous over SS and $\mathrm{Co}-\mathrm{Cr}-\mathrm{Ni}$ coil springs for clinical application and showed the least percentage of force degradation. For this reason a Nitinol closed coil springs were used in current study. Comparison of each time (T2-T4) with the baseline (T1) in both groups demonstrated significant elevation in IL-2 level at the control group one week after baseline (T2), while the elevation of IL-2 level that took place at the test group wasn't significant owing this to the larger variation seen between individuals at the test group more than at the control group. This inter or even intra-individual differences in tooth movement characteristics are probably linked to individual difference in the structure of and cellular activity within the PDL, alveolar bone, and localized differences in the expression of factors such as growth factors and growth cytokines. ${ }^{7}$

The increase in the level of IL-2 in both groups one week after applying the retraction forces could be linked to the initial tooth movement phase which involves an acute inflammatory reaction, characterized by periodontal vasodilation and different cells migration to the area of inflammation. Migratory cells release numerous cytokines, which directly or indirectly interact with the entire population of native para-dental cells to enhance orthodontic tooth movement. At (T3) the statistically substantial increase was seen at the test group, which could be due to overlaying acute inflammation on ongoing chronic inflammation which caused significant promotion in the concentration of inflammatory cytokines, ${ }^{25}$ such as IL-2, while at the control group the IL-2 level almost returned to baseline, which might be due to a refractory period, ${ }^{26}$ decrease number of IL-2 positive cells and their association with bone, this suggests that IL-2 level might not return to baseline when the forces are incrementally increased weekly by preventing the occurrence of the refractory period at the test group. At (T4) both groups showed a significant increase in IL-2 level, which was more significant at the test group. Hence our deduction from above findings is that weekly increase in retraction forces could provide more effective up-regulation of IL-2 levels than the conventional force which tends to decay over the fortnight period. This information further translates into that weekly activation prevents the refractory period which essentially slows down the space closure process. Therefore, the efficiency of space closure is compromised. Finally, the alternative hypothesis can be accepted that there is a significant increase in IL-2 levels with gradual increasing force during space closure in Orthodontics.

A recent publication is highlighting the other aspect of biomarkers such as alkaline phosphatase activity enhanced during increased incremental forces in bone deposition on the tension side during orthodontic treatment. ${ }^{27}$ Future studies would aim to explore tight regulations or feedback mechanisms that prevent these mediators from increasing excessively, considered indispensable to avoid any harmful consequences. For example, excessive bone resorption molecule such as local prostaglandin E2 (PGE2) concentration leads to iatrogenic root resorption in rats, ${ }^{28}$ or in humans. ${ }^{29}$ If this is the case, it remains to be determined how to maximize cellular response while not exceeding a certain threshold, to achieve faster tooth movement. Interestingly future orthodontic case by case assessment would perhaps utilize individualized biomarker evaluation to provide best optimum force delivery for each case.

\section{Conclusion}

This study using a split mouth technique attempted to determine the effect of using different types of orthodontic forces (relatively constant versus gradually increasing) on the level of interleukin 2 (a biomarker reflecting the process of bone resorption) during maxillary canine retraction. The sample consisted on ten participants selected randomly from the orthodontic department of Alexandria University according to specific criteria. Maxillary first premolars were extracted from all participants and the phase of leveling and alignment was completed. A relatively constant force side and a gradually increasing force side were assigned randomly to the right and left sides of the maxillary arch. For each participant, the maxillary canines receiving the relatively constant force were considered to be the control group and were subjected to a continuous retraction force of $150 \mathrm{~g}$ for three weeks, while the contralateral canines receiving the gradually increasing retraction forces were considered to be the test group, and they received an initial force of $75 \mathrm{~g}$ at the first week, followed by $150 \mathrm{~g}$ at the $2 \mathrm{nd}$ week and finally $300 \mathrm{~g}$ at the $3 \mathrm{rd}$ and last week.

Gingival crevicular fluid was collected using paper strips from each participant at baseline (T1), 1week after baseline (T2), 2weeks 
after baseline (T3) and 3 weeks after baseline (T3). Enzyme-linked immunoassay kit was used to measure IL-2 level in the GCF.The data obtained from this study were analyzed using Wilcoxon signed ranks test was used. Our study showed that by weekly increasing the retraction forces on maxillary canines we could achieve more effective up-regulation of IL-2 level than using weekly relative constant level of force and thus achieving more efficiency on inducing initial tissue reaction for bone resorption.

\section{Acknowledgment}

Above all, utmost appreciation to the Almighty Allah for the divine intervention in this academic endeavor; Sincere gratitude to my good friend Prof. Shazia Naser-Ud-Din for her engorgement, contribution and efforts in order for this study to be published

\section{Conflict of interest}

I declare that no financial interest or any conflict of interest exists.

\section{Funding}

None

\section{References}

1. Muira F, Mogi M, Ohura Y, et al. The Superelastic Japanese NiTi alloy wire for use of Orthodontics. Part III. Studies on the Japanese NiTi alloy coil spring. Am J Orthod Dentofacial Orthop. 1988;94(2):89-96.

2. McLaughlin RP, Bennett JC. Evolution of treatment mechanics and contemporary appliance design in orthodontics: A 40 year perspective. Am J Orthod Dentofacial Orthop. 2015;147(6):654-662.

3. Rygh P, Brudvik P. The histological responses of the periodontal ligament to horizontal orthodontic loads. In: Berkovitz BB, Moxham BJ \& Newman HN (Eds.), The periodontal ligament in health and disease. Mosby, St Louis,USA:1995.

4. Davidovitch Z. Tooth movement. Crit Rev Oral Biol Med. 1991;2(4):411450.

5. Melsen B. Biological reaction of alveolar bone to orthodontic tooth movement. Angle Orthod. 1999;69(2):151-158.

6. Proffit WR. Biologic basis of orthodontic therapy. In: Proffit WR \& Fields HW (Eds.), Contemporary orthodontics. Mosby, St Louis, USA: 2000.

7. Ren Y, Maltha JC, Kuijpers-Jagtman AM. Optimum force magnitude for orthodontic tooth movement-a systematic review. Angle Orthod. 2003;73(1):86-92.

8. Kapoor P, Kharbanda OP, Monga N, et al. Effect of orthodontic forces on cytokine and receptor levels in gingival crevicular fluid: a systematic review. ProgOrthod. 2014;15:65.

9. Charles H. A clinical investigation of the concepts of differential and optimal force in canine retraction. Am J Orthod. 1974;44(2):113-119.

10. Cannon JG. Inflammatory Cytokines in Nonpathological States. News Physiol Sci. 2000;15:298-303.

11. Stoycheva MS, Murdjeva MA. Correlation between serum levels of interleukin 1 , interleukin-6, interleukin 10 , interleukin 12 , tumor necrosis factor and interferon- with some clinical and laboratory parameters in patients with salmonellosis. Biotechnol Equip. 2005;19:143-146.
12. Scarel-Caminaga RM, Trevilatto PC, Souza AP, et al. Investigation of an IL-2 polymorphism in patients with different levels of chronic periodontitis. J Clin Periodontol. 2002;29(7):587-591.

13. Thornton AM, Shevach EM. CD4+CD25+ immunoregulatory T cells suppress polyclonal $\mathrm{T}$ cell activation in vitro by inhibiting interleukin 2 production. J Exp Med. 1998;188(2):287-296.

14. Stern J, Smith KA. Interleukin-2 induction of T-cell G1 progression and c-myb expression. Science. 1986;233(4760):203-206.

15. Basaran G. Interleukins 2, 6, and 8 levels in human gingival sulcus during orthodontic treatment. Am J Orthod and Dentofacial Orthop. 2006;130(1):7.e1-7.e6.

16. Grant M, Wilson J, Rock P, et al. Induction of cytokines, MMP9, TIMPs, RANKL and OPG during orthodontic tooth movement. Eur $J$ Orthod. 2013;35(5):644-651.

17. Insoft M, King GJ, Keeling SD. The measurement of acid and alkaline phosphatase in gingival crevicular fluid during orthodontic tooth movement. Am J Orthod Dento facial Orthop. 1996;109(3):287-296.

18. Grieve W, Johnson G, Moore R, et al. Prostoglandin E (PGE2) and Interleukin-1 $\beta$ (IL-1 $\beta$ ) levels in gingival crevecular fluid during human orthodontic tooth movement. Am J Orthod Dento facial Orthop. 1994;105(4):369-374.

19. Uematsu S, Mogi M, Deguchi T. Interleukin (IL)-1 $\beta$, IL-6, tumor necrosis factor, epidermal growth factor and $\beta 2$ micro globulin levels are elevated in gingival crevicular fluid during human orthodontic tooth movement. $J$ Dent Res. 1996;75(1):562-567.

20. Alhashimi N, Frithiof L, Brudvik P, et al. CD 40-CD 40L expression during orthodontic tooth movement in rats. Angle Orthod. 2004;74(1):100-105.

21. Reitan K. Tissue behavior durin $\backslash$ g orthodontic tooth movement. Am J Orthod. 1960;46(12):881-890.

22. Cuida M, Brun JG, Tynning T, et al. "Calprotectin levels" in Neurotransmitters, cytokines and the control of alveolar bone remodeling in orthodontics. Dent Clin North Am. 1988;32(3):411-435.

23. Serra E, Perinetti G, D'Attilio M, et al. Lactate dehydrogenase activity in gingival crevicular fluid during orthodontic treatment. Am J Orthod Dentofacial Orthop. 2003;124(2):206-211.

24. Angolkar PV, Arnold JV, Nanda RS, et al. Force degradation of closed coil springs. Am J Orthod and Dentofacial Orthop. 1992;102(2):127-133.

25. Ren Y. Cytokine levels in crevicular fluid are less responsive to orthodontic force in adults than in juveniles. J ClinPeriodontol. 2002;29(8): 757-762.

26. Smith KA. Interleukin-2: inception, impact, and implications. Science. 1988;240(4856):1169-1176.

27. AlSwafeeri H, ElKenany W, Mowafy M, et al. Crevicular alkaline phosphatase activity during the application of two patterns of orthodontic forces. J Orthod. 2015;42(1):5-13.

28. Leiker BJ, Nanda RS, Currier GF, et al. The effects of exogenous prostaglandins on orthodontic tooth movements in rats. Am J Orthod Dentofacial Orthop. 1995;108(4):380-388.

29. Lee KJ, Park YC, Yu HS, et al. Effects of continuous and interrupted orthodontic force on interleukin-1 $\beta$ and prostaglandin E2 production in gingival crevicular fluid. Am $J$ Orthod Dentofacial Orthop. 2004;125(2):168-177. 